\title{
4D-STEM Characterization of Molecular Ordering in Organic Semiconductors
}

\author{
Gabriel Calderon ${ }^{1}$, Letian Dou ${ }^{2}$ and Jinwoo Hwang ${ }^{1}$
}

1. Department of Materials Science and Engineering, The Ohio State University, Columbus, OH, USA.

2. Davidson School of Chemical Engineering, Purdue University, Lafayette, IN, USA.

* Corresponding author: hwang458@osu.edu

The nanoscale ordering amongst the constituent molecules plays a crucial role in determining the electronic, optical, and photovoltaic properties of organic semiconductors [1]. Understanding of the molecular ordering requires characterization methods that can precisely reveal the details of the ordering. In some cases, such as in TFT-LCD, where the degree of ordering is extremely high and the molecules are almost uniformly semi-crystalline, a large area diffraction technique (e.g. X-ray diffraction) can provide fairly good information about the ordering. However, in many cases, such as in organic photovoltaic (OPV) thin films, the ordering is much less in degree and more subtle in detail, which makes it much more difficult to characterize using any conventional diffraction or imaging methods. This "gap in the characterization" and the consequent challenges in understanding the important structure-property relationships have been the major obstacles in the development of new-generation OPV materials and devices for energy harvesting applications.

We perform 4-dimensional scanning transmission electron microscopy (4D-STEM) using a fast pixel array STEM detector [2], combined with fluctuation microscopy based on electron nanodiffraction [3] to investigate the nanoscale molecular ordering in OPV thin films. A couple of obstacles for this approach are the radiation damage and contamination of the sample inside TEM. We found that the two issues are in fact related, both originated from the residual solvent (chlorobenzene) that we use when we spin coat the material, the P3HT polymers. Since conventional plasma cleaning is unusable because it destroys the polymer molecule itself, we used the in situ vacuum annealing to remove the residual solvent, which in turn greatly helped reduce both radiation damage and contamination of the film (Figure 1).

Scanning the electron probe (size $\sim 1 \mathrm{~nm}$ ) over the OPV sample generates nanodiffraction patterns with spatial oversampling, which are then captured at the rate of $1 \mathrm{msec}$ per frame using the Thermofisher EMPAD detector (Figure 2a). Although the scattering intensity is weak due to the low $Z$ number, the distinct diffraction speckles (Figure $2 b$ ) suggest that the patterns do capture the spatially resolved ordering signal. The diffraction patterns were used to calculate the normalized variance, $V$, as a function of $k$, the scattering vector. $V(k)$ shows multiple peaks, each of which relates to a specific ordering type (Figure 2c). The peak amplitude shows the relative degree of the ordering. We found that the degree of ordering, particularly the (200) ordering of the molecules, increases when a higher RPM is used in spin coating, suggesting that more anisotropy induced by the spinning force can enhance the ordering.

The nanodiffraction patterns can then be reconstructed into the spatial maps of the ordering, both as a function of $k$ (type) and orientation, $\varphi$ (Figure 3 ). These multi-dimensional maps can provide significantly more information about the ordering as compared to the conventional methods, including the size, distribution, percolation, and volume fraction of the molecular ordering at the nanoscale. This new direct structural information can then provide crucial inputs to the theory and simulation (e.g. [4]) to establish exact relationship between the molecular ordering and important properties of OPV films. 
References:

[1] R Noriega et al., Nature Materials 12 (2013), p. 1038.

[2] M Tate et al., Microscopy and Microanalysis 22 (2016), p. 237.

[3] J Hwang et al., Physical Review Letters 108 (2012), p. 195505.

[4] S Mollinger et al., ACS Macro Letters 4 (2015), p. 708.

(a)
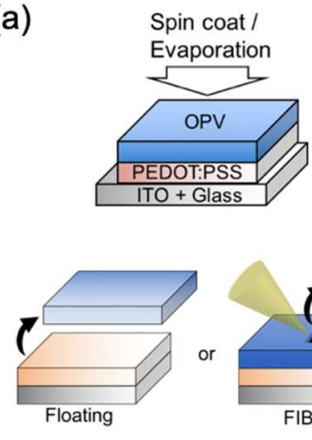

Plan view

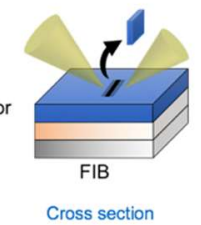

(b)

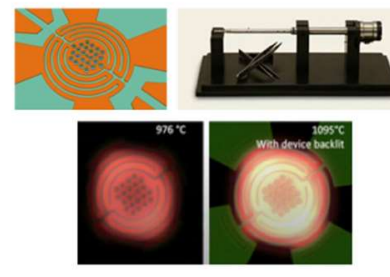

In situ annealing in TEM $130^{\circ} \mathrm{C}$ for an hour (c)
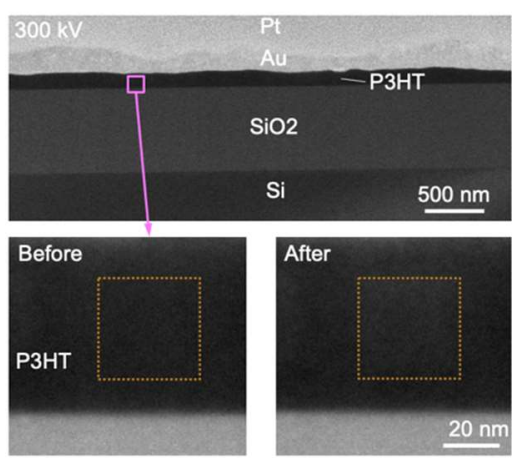

Figure 1. (a) TEM sample preparation of OPV films, both in plan view and cross section. (b) In situ annealing setup. (c) A cross section view of the sample in STEM, showing marginal signs of radiation damage and contamination after the beam scan.

(a)

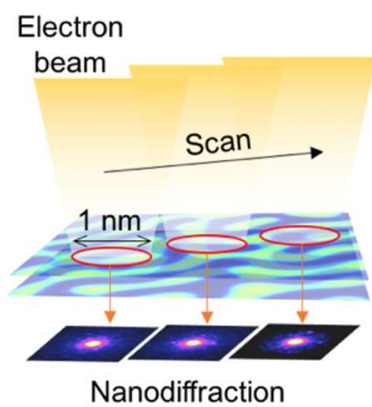

(b)

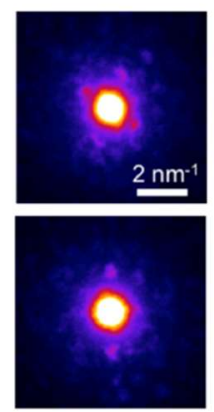

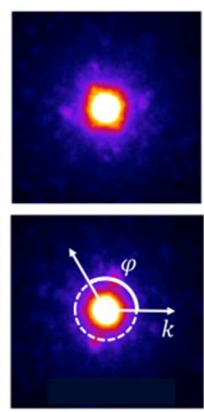

(c)

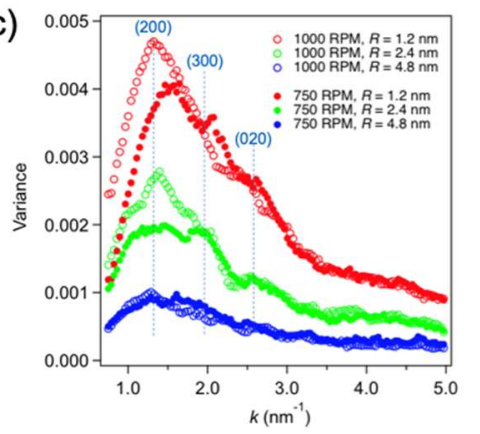

Figure 2. (a) 4D-STEM scan of the probe over OPV film. (b) Nanodiffraction patterns from P3HT, which are analyzed to yield the $V(k)$ data shown in (c). The data shows that the degree of ordering increases as more anisotropy is induced to the film by increasing the spin coating RPM.

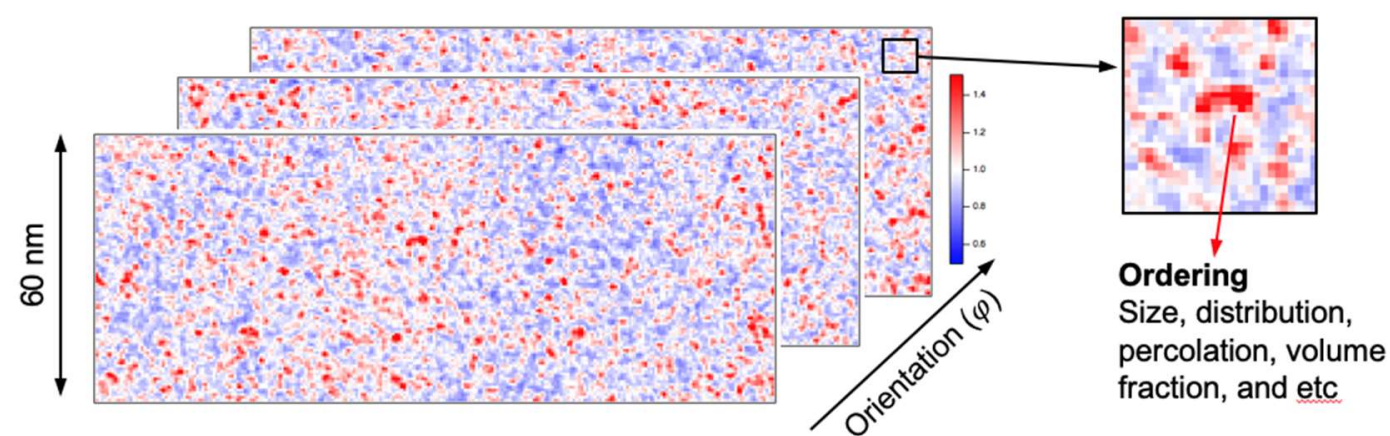

Figure 3. The spatial maps of the molecular ordering as a function of the in-plane orientation angle reconstructed using the 4D-STEM data. The parameters of the ordering can be directly determined from the maps, including size, distribution, percolation, and volume fraction of the ordered regions. 